INRA Prod. Anim., 2002, 15 (3), 221-230
J. J. COLLEAU ${ }^{1}$, P. QUÉMÉRÉ ${ }^{2}$, H. LARROQUE ${ }^{1}$, J. SERGENT ${ }^{3}$, C. WAGNER ${ }^{4}$

${ }^{1}$ INRA Station de Génétique Quantitative et Appliquée, 78350 Jouy-en-Josas cedex

${ }^{2}$ Institut Supérieur d'Agriculture de Beauvais, rue Pierre Waguet,BP 313 , 60026 Beauvais cedex

${ }^{3}$ Parc Naturel Régional d'Armorique, 15 place aux foires, 29590 Le Faou

${ }^{4}$ Société des éleveurs de la race bovine Bretonne Pie-Noire, allée Sully, 29322 Quimper cedex

Courriel : ugencjj@dga2.jouy.inra.fr

\section{Gestion génétique de la race bovine Bretonne Pie-Noire : bilan et perspectives}

Si au début du $20^{\text {ème }}$ siècle l'effectif de la race Bretonne Pie-Noire atteignait encore un demi-million de têtes, il ne restait plus que 15000 femelles en 1975. Un programme de conservation de cette race, le premier appliqué à l'espèce bovine, a alors été mis en place. Son objectif : conserver au maximum la diversité génétique des individus tout en maîtrisant l'augmentation de la consanguinité.

Lintensification de l'agriculture européenne et particulièrement française s'est accompagnée à partir des années 1960-1970 d'une désaffection généralisée pour un certain nombre de races et populations bovines, jugées peu productives. Cependant, des voix se sont élevées pour signaler le risque d'atteinte au patrimoine génétique bovin qu'entraînerait une indifférence totale de la société face à ces phénomènes (Vissac 1972, Bougler 1974, Audiot 1995). C'est la raison pour laquelle au milieu des années 70, des programmes de conservation se sont mis en place avec l'aide officielle du ministère de l'Agriculture puis du Bureau des Ressources Génétiques (depuis 1983), ainsi que la participation effective de divers organismes régionaux et nationaux. Le programme de conser-

\section{Résumé}

\footnotetext{
La race bovine Bretonne Pie-Noire est l'une des douze races bovines actuellement en conservation en France. Son programme de sauvegarde remonte à 1976. Après avoir rappelé le contexte socio-économique correspondant à cette race et l'évolution des effectifs bovins impliqués, l'article présente les principes de la gestion génétique du plan de sauvegarde appliqués jusqu'en 2000 et les évolutions des critères habituels de variabilité génétique (parenté, consanguinité, nombre de lignées génétiques représentées). On présente les motivations et principes d'un nouveau plan de sauvegarde, qui devrait être plus efficace que l'ancien. Une extrapolation des tendances passées montre que ce dernier aurait généré en 2030 des coefficients de consanguinité moyens de l'ordre de 10-11 \%. Des simulations du nouveau plan montrent qu'à la même date ces coefficients moyens seraient seulement de l'ordre de 4,5 à $5 \%$.
}

vation de la race Bretonne Pie-Noire date de cette époque (1976) et est le premier programme appliqué aux bovins. Il fait suite à une enquête réalisée sur le terrain en 1975 (Quéméré et Bertrand 1976), auprès de 230 éleveurs et 49 responsables techniques divers, par une équipe d'enseignants et d'étudiants de l'Institut Supérieur d'Agriculture de Beauvais (ISAB).

A intervalles réguliers, des bilans de ce programme ont été publiés (Quéméré et al 1978, 1981, 1992 et 1999) qui se sont surtout attachés à décrire l'évolution du contexte socioéconomique de la microsociété des éleveurs concernés, à exposer les problèmes spécifiques liés à l'animation de tels schémas et à dégager des voies d'amélioration. La description initiale de la gestion génétique (Colleau et al 1976) n'a pas été suivie de documents publics ultérieurs. L'objet de cet article est précisément de combler cette lacune en dressant le bilan détaillé de la gestion génétique du plan de conservation depuis plus de 20 ans. Pour une meilleure compréhension des principes d'action correspondants, le contexte socio-économique et son évolution dans le temps seront brièvement rappelés. On exposera les principes et le bilan de la gestion génétique de la population, à partir de la mise en place du plan jusqu'au présent. Pour terminer, les motivations et modalités du nouveau plan initié en 2001 seront présentées. 


\section{1 / Le contexte}

\section{1 / Caractéristiques de la race}

La race Bretonne Pie-Noire (BPN) est originaire de la Cornouaille et du pays de Vannes (Finistère-Sud et Morbihan). Son rameau PieRouge, localisé surtout dans le Léon et le Trégor, a disparu au début du siècle à la suite des croisements avec la Durham pour aboutir à la race Armoricaine, elle aussi engagée dans un programme de conservation. La vache BPN est de petit format ( poids adulte de 350$450 \mathrm{~kg}$ ) avec, à la fin des années 90 , une production par lactation de l'ordre de $3500 \mathrm{~kg}$ et une composition du lait qui rappelle celle de la Normande (44 g/kg de taux butyreux et $34 \mathrm{~g} / \mathrm{kg}$ de taux protéique). Au moment de l'enquête de 1975, les effectifs n'étaient plus que d'environ 15000 femelles, avec un taux de renouvellement très faible $(2 \%)$. On peut se demander ce qu'il serait advenu de la race si le plan de sauvegarde n'avait pas existé. En effet, la presque totalité des 1000 femelles BPN actuelles est intégrée au plan de sauvegarde. Au début du $20^{\text {ème }}$ siècle, il y avait encore environ $500000 \mathrm{BPN}$. Son format, ses faibles besoins ainsi que sa relative productivité rendaient l'animal BPN adapté à l'agriculture bretonne de l'époque : sur des terres pauvres, à vocation céréalière pour l'alimentation humaine, la vache laitière valorisait les terrains marginaux, assurait l'autoapprovisionnement en produits laitiers et amendait les sols grâce à son fumier.

Les auteurs du $19^{\text {ème }}$ siècle (Sanson 1884) considéraient la BPN comme apparentée à la race irlandaise Kerry, elle aussi de petit format. La comparaison avec les races françaises grâce à la mesure des polymorphismes et distances génétiques (Grosclaude et al 1990) révèle plutôt une parenté avec le groupe des races de l'Est (Vosgienne, Montbéliarde, Pie-Rouge de l'Est, Abondance, Tarine). Ce gradient Sud-Est Nord-Ouest est, d'après ces auteurs, conforme à divers travaux d'archéozoologie qui indiqueraient que l'introduction de bovins à cornes courtes en Europe s'est faite par le Sud-Est, en provenance d'Asie Mineure et de Mésopotamie.

\section{2 / Contexte socio-économique}

Les premiers éleveurs engagés dans le programme en 1976, au nombre de 46 et déten- teurs de seulement 311 vaches sur les 15000 existantes, étaient essentiellement intéressés par l'aspect conservatoire du plan, moyen d'empêcher l'extinction complète d'une race à laquelle ils étaient encore attachés, même s'ils exploitaient aussi des animaux des races les plus usitées et vendaient leur lait aux laiteries. Ces éleveurs étaient très dispersés sur 5 départements.

Une enquête effectuée 15 ans après (Henry 1992, Quéméré et al 1992) révélait que ce type d'éleveurs ne représentait plus que $30 \%$ d'entre eux. Une moitié était alors constituée d'ardents défenseurs de la race, qu'ils considéraient comme un élément essentiel d'un système de transformation fermière, avec une bonne valorisation des produits, laitiers principalement. Les $20 \%$ restant (retraités, pluriactifs et rurbains) concevaient l'élevage en amateurs passionnés et utilisaient la BPN comme une race allaitante en très petit effectif.

Le titre de l'enquête de Brossard en 1999 ("Vers une relance de la race bovine Bretonne Pie-Noire') montre bien le changement de climat (Quéméré et al 1999). En effet, il se confirme que les 'idéalistes-transformateurs' ont réussi à mettre au point des systèmes économiques crédibles avec vente directe de produits très diversifiés. L'orientation Agriculture Biologique concerne en outre une bonne moitié des éleveurs correspondants. Sachant que la société française en général prône maintenant le retour à un minimum d'écologie des pratiques agricoles, de respect de l'environnement et d'authenticité des produits, il est logique que l'enquête conclue à une bien meilleure perception de la BPN en dehors de son microcosme : elle n'est plus une race témoin du passé, mais postule à un certain avenir.

Il convient de noter que cette dernière période confirme aussi l'extension des élevages secondaires privés (amateurs, pluriactifs, agrotourisme) ou publics (écomusée, collectivités territoriales pour entretien d'espaces verts) qui représentent alors $80 \%$ des éleveurs et détiennent environ $55 \%$ du cheptel. Ils se fournissent à partir des troupeaux laitiers qui, dès lors, n'hésitent plus à élever un maximum de génisses afin de les tester en production. On note également que la dispersion géographique augmente : en 2000, les BPN du plan se situent dans 19 départements au lieu des 5 initiaux. Le tableau 1 donne une

Tableau 1. Statistiques générales sur la population femelle du programme de conservation de la race Bretonne Pie-Noire.

\begin{tabular}{|l|c|c|c|c|c|c|c|}
\hline Année & 1976 & 1980 & 1984 & 1988 & 1992 & 1996 & 2000 \\
Nombre d'éleveurs & 46 & 47 & 49 & 72 & 96 & 192 & 270 \\
Nombre d'éleveurs au contrôle laitier & 5 & 8 & 9 & 14 & 9 & 12 & 13 \\
Nombre d'éleveurs avec plus de 10 vaches & 8 & 10 & 15 & 18 & 19 & 20 & 24 \\
Nombre d'éleveurs avec moins de 4 vaches & 23 & 25 & 26 & 35 & 70 & 169 & 200 \\
\hline Nombre de vaches & 277 & 250 & 272 & 324 & 345 & 530 & 700 \\
Nombre de vaches au contrôle laitier & 60 & 90 & 112 & 144 & 101 & 125 & 125 \\
Nombre de génisses & 34 & 160 & 136 & 135 & 203 & 231 & 300 \\
Effectif total & $\mathbf{3 1 1}$ & $\mathbf{4 1 0}$ & $\mathbf{4 0 8}$ & $\mathbf{4 5 9}$ & $\mathbf{5 4 8}$ & $\mathbf{7 6 1}$ & $\mathbf{1 0 0 0}$ \\
Effectif moyen par éleveur & 6,8 & 8,7 & 8,3 & 6,3 & 5,7 & $\mathbf{4 , 0}$ & 3,7 \\
\hline
\end{tabular}


synthèse chiffrée de cette évolution depuis bientôt 25 ans, à partir des informations internes provenant de la gestion du programme (fichiers Société des éleveurs-INRA).

On retrouve les faits mentionnés précédemment : à partir du début des années 1990 , explosion du nombre d'éleveurs mais diminution de la taille moyenne des élevages. Lautre fait marquant est la croissance de la population qui double pratiquement de taille au cours des 10 dernières années.

Les modalités d'encadrement et de financement du programme de conservation sont décrites dans l'encadré 1.

\section{2 / Bilan du plan 1977-2000}

\section{1 / Principes du programme de conservation}

Lobjectif du programme de conservation était bien entendu de conserver le maximum de lignées génétiques présentes dans la population de départ en produisant un minimum de consanguinité, celle-ci traduisant à la fois une réduction de l'éventail génétique et pouvant affecter directement la viabilité et les performances des animaux.
Au départ, on disposait de 22 mâles de généalogie connue, dont on possédait encore de la semence congelée ou utilisés chez les éleveurs ayant souscrit un contrat. Ces mâles ont été répartis en 8 groupes avec un fort apparentement intra groupe et un faible apparentement entre groupes.

Suivant l'approche en usage à l'époque (décrite par De Rochambeau et al 2000), les femelles ont été réparties dans un même nombre de groupes suivant le même principe. La disposition initiale des groupes sur un cercle (figure 1) était telle que les familles étaient d'autant plus éloignées qu'elles étaient apparentées.

Par la suite, à intervalles réguliers, le schéma d'accouplement était modifié suivant un système préétabli. L'anneau' des mâles tournait d'un cran tous les 2 ans. Les 3 mâles de départ dont le stock de semence était très limité ont été remplacés en priorité. On prévoyait ensuite modestement d'introduire un nouveau mâle tous les 2 ans (cf les contraintes énoncées plus haut), issu de l'accouplement d'une vache présentant un minimum de production au contrôle laitier et du taureau à remplacer. Les recommandations pour la gestion du troupeau femelle étaient de retarder au maximum la réforme et de tenter le remplacement de chaque vache par une fille.

\section{Encadrement et financement du programme de conservation} de la race Bretonne Pie-Noire

L'encadrement local est indispensable pour assurer le minimum de suivi zootechnique et généalogique dans les élevages conformément au programme de conservation et pour animer la Société des éleveurs (gestion courante et promotion de la race). La Société de éleveurs de Bretonne PieNoire, société reconstituée en 1975 après une disparition effective depuis 1972, est maître d'œuvre du programme.

Les débuts ont été difficiles : cet encadrement a été effectué d'abord par des étudiants stagiaires (M. Le Borgne, 1977) puis à temps partiel par des agents du Contrôle Laitier, L. Collin (1980-1983) et C. Goenvec (1983-1988) ou par des agents externes, L. Flageul (19891990). Depuis 1990, la situation est plus satisfaisante puisque le Parc Naturel Régional d'Armorique (PNRA) participe à la sauvegarde des races domestiques bretonnes. Un technicien de cet organisme (J. Sergent depuis 1990) consacre $2 / 3$ de son temps à l'animation technique du programme de sauvegarde de la race Bretonne Pie-Noire $(\mathrm{BPN})$. De plus, le PNRA produit des génisses amouillantes pour satisfaire une demande croissante en sujets de la race, liée à l'installation de nouveaux éleveurs (une soixantaine de sujets vendus depuis 1993). Il élève enfin des reproducteurs mâles avant le prélèvement de semence par les centres d'insémination artificielle.

P. Quéméré (ISAB), initiateur de l'enquête de situation, assure depuis le début l'animation générale.
T. Krychowski (UNLG) en 1976 puis J-J. Colleau (INRA) assurent le suivi du programme génétique. La gestion des fichiers zootechniques, à la suite des tournées annuelles de l'animateur local, est initiée au PNRA puis complétée à l'INRA (M. Dupont puis H. Larroque depuis 1994).

Les aides financières directes proviennent du ministère de l'Agriculture, de la région Bretagne, des différents départements bretons et celui de Loire-Atlantique. Ces aides permettent de prendre en charge les frais de fonctionnement de la Société des éleveurs ainsi que le programme d'action technique d' élevage. Elles visent à favoriser l'élevage des génisses déclarées à l'état-civil (500F, soit environ 76 euros, par animal), l'incitation au contrôle laitier (remboursement à $85 \%$ ), l'insémination artificielle (remboursement total) et l'élevage des petits mâles, candidats au remplacement des taureaux d'insémination artificielle (1000 F, soit environ 152 euros, par animal).

Une aide indirecte est apportée par le PNRA qui prend en charge le salaire de l'animateur local et par les unités de sélection de l'Ouest (URCEO et OGER). Le nombre de taureaux entrant annuellement dans le programme étant faible (de l'ordre de 1), cellesci assurent gratuitement la récolte de semence, qui est aussi partiellement utilisée pour les inséminations artificielles de BPN sur d'autres races (à la fin des années 90, le nombre annuel d'inséminations artificielles avec des taureaux BPN, en race pure ou en croisement, est d'environ 2000). 
Figure 1. Principe du plan d'accouplement.

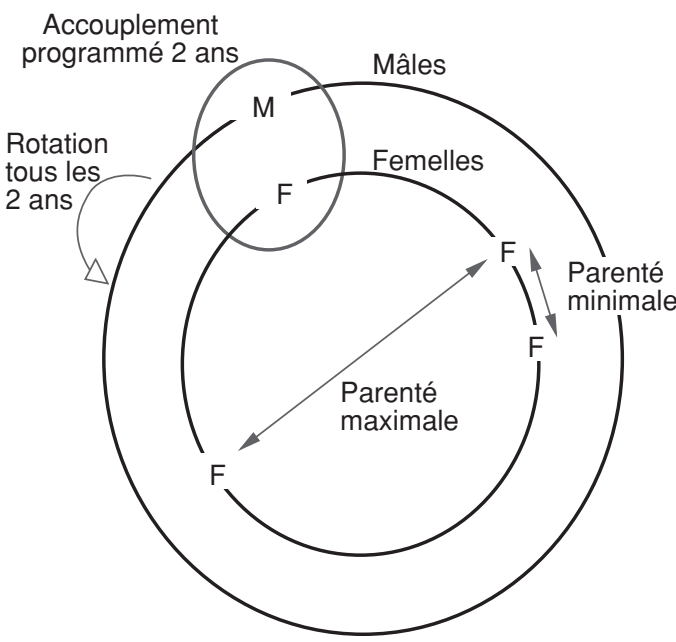

\section{Consanguinité et expression des tares héréditaires}

Imaginons une population de 900 vaches dans laquelle existe une maladie génétique, contrôlée par un gène récessif et telle que les homozygotes pour le mauvais allèle meurent à la naissance (en présentant éventuellement des malformations spectaculaires). Supposons que la fréquence du mauvais allèle soit $p=1 / 30$.

Si les veaux ne sont pas consanguins, l'espérance $\mathrm{Na}$ du nombre de veaux affectés est alors égale à $900 p^{2}$ c'est-à-dire 1 . S'ils présentent un taux de consanguinité de $\mathrm{F}$ (valeur comprise entre 0 et 1 ), on a alors

$$
\mathrm{N}_{\mathrm{a}}=900\left[\mathrm{~F} p+(1-\mathrm{F}) p^{2}\right]
$$

Pour $\mathrm{F}=0,02 ; 0,05 ; 0,10$ on trouve alors que $\mathrm{N}_{\mathrm{a}}$ devient 1,$6 ; 2,5 ; 3,9$ respectivement. La valeur $\mathrm{F}=0,05$ qui a été prise comme référence à ne pas dépasser fait déjà plus que doubler le risque de base (celui qu'on aurait sans consanguinité).
Dans ces conditions, on escomptait une progression annuelle du taux de consanguinité de l'ordre de $0,17 \%$. Les taux de consanguinité élevés se traduisent par des pertes de variabilité génétique, des performances altérées et des risques accrus d'expression de tares héréditaires. En considérant surtout ces derniers (voir encadré 2), on peut estimer qu'il est préférable de se situer toujours en deçà d'une valeur de $5 \%$. Cette zone critique devant normalement être atteinte vers 20052010 ; il était a priori évident que ce plan devait être remanié dans le long terme soit par changement de conception, soit par mise en ouvre de moyens plus puissants, soit les deux à la fois.

\section{2 / Gestion effective des pères}

Le tableau 2 montre la situation concernant l'utilisation des pères. On notera la présence d'une fraction non négligeable de taureaux de monte naturelle (l'existence de ce fait est prise en considération dans le nouveau plan) qui ont contribué à augmenter le nombre absolu de taureaux : celui-ci a varié de 15 en 1978 à 30 en 1999, valeurs différentes du 8 du schéma initial. Une durée moyenne d'utilisation de 10 ans (et non de 16) a été observée pour les taureaux d'IA qui ne sont plus en service.

\section{3 / Paramètres démographiques de la population femelle}

Le tableau 3 montre la croissance rapide des effectifs de génisses ce qui donne lieu à un rajeunissement de la pyramide des âges des reproductrices. De toute évidence, étant donné le rajeunissement des mères à la naissance des génisses, la procréation de ces dernières commence à s'effectuer très tôt dans la vie. Cette démographie traduit le fait que la conduite générale n'est plus une conduite de sauvegarde mais déjà une conduite de relance, avec une certaine confiance en l'avenir : les raisons en ont été évoquées plus haut.

Sous l'hypothèse d'une constance de ces paramètres démographiques, l'analyse de l'ensemble des cohortes de génisses initiées dans le cadre du plan de conservation permet d'aboutir au déroulement de carrière indiqué au tableau 4. La durée moyenne de la carrière

Tableau 2. Statistiques générales sur la gestion des pères en fonction de l'année de naissance des filles. $I A$ : insémination artificielle, $M N$ : monte naturelle.

\begin{tabular}{|l|c|c|c|c|c|c|}
\hline \multicolumn{1}{|c|}{ Année de naissance } & 1978 & 1982 & 1986 & 1990 & 1994 & 1999 \\
\hline Nombre de génisses & 86 & 59 & 72 & 89 & 105 & 130 \\
\hline Nombre de pères IA & 7 & 12 & 9 & 12 & 14 & 10 \\
\hline Nombre de pères MN & 8 & 10 & 15 & 18 & 19 & 20 \\
\hline \% filles issues d'IA & 83 & 97 & 90 & 79 & 73 & 86 \\
\hline Age des pères (années) & 13,0 & 10,3 & 12,6 & 10,7 & 8,1 & 12,3 \\
\hline
\end{tabular}

Tableau 3. Age des mères en fonction de l'année de naissance des filles.

\begin{tabular}{|l|c|c|c|c|c|c|}
\hline Année de naissance & 1978 & 1982 & 1986 & 1990 & 1994 & 1999 \\
\hline Nombre de génisses & 86 & 59 & 72 & 89 & 105 & 130 \\
Age des mères (années) & 7,9 & 6,4 & 5,5 & 6,0 & 5,3 & 5,2 \\
\hline
\end{tabular}


Tableau 4. Déroulement de carrière des vaches du plan (devenir d'une cohorte de vaches nées la même année).

\begin{tabular}{|c|c|c|}
\hline $\begin{array}{c}\text { Années } \\
\text { observées }\end{array}$ & $\begin{array}{c}\text { \% de vaches } \\
\text { survivantes }\end{array}$ & $\begin{array}{c}\text { Nombre de } \\
\text { filles par vache } \\
\text { de départ }\end{array}$ \\
\hline 3 & 100 & 0,22 \\
4 & 89,1 & 0,42 \\
5 & 77,8 & 0,60 \\
6 & 68,6 & 0,74 \\
7 & 60,0 & 0,86 \\
8 & 53,1 & 0,96 \\
9 & 46,5 & 1,03 \\
10 & 39,7 & 1,08 \\
11 & 35,0 & 1,14 \\
12 & 30,4 & 1,19 \\
13 & 25,5 & 1,21 \\
14 & 20,1 & 1,23 \\
15 & 14,9 & 1,26 \\
16 & 12,6 & 1,27 \\
17 & 8,6 & 1,28 \\
18 & 4,4 & 1,28 \\
19 & 2,9 & 1,29 \\
20 & 1,5 & 1,29 \\
\hline
\end{tabular}

est de 8,7 ans. Le tableau 4 indique que dans une cohorte de vaches observée sur 8 ans ou 9 ans, chaque vache laisse en moyenne 0,96 ou 1,03 fille respectivement. La durée moyenne de la carrière étant de 8,7 ans, on serait tenté de croire que chaque vache laisse environ 1 fille. Il n'en est rien puisque certaines vaches vivent plus longtemps que 8 ou 9 ans et continuent à produire des filles. A l'extinction de la cohorte au bout d'environ 20 ans, on trouve finalement qu'une vache moyenne est remplacée par 1,29 fille.

\section{4 / Qualité des généalogies}

Les animaux initiaux du plan ont pu bénéficier de l'essentiel du fichier des généalogies tenues par l'ancien Herd-Book (jusqu'en 1972, date de sa disparition momentanée). Par la suite, la croissance des effectifs s'est faite à partir de ces animaux 'originels'. Fin 1999, le fichier généalogique comprenait 3831 individus, dont le plus ancien était né en 1910 (24 générations au maximum). Ces faits expliquent la bonne qualité des généalogies, appréciée par le nombre moyen d'équivalents-générations (tableau 5).

La définition de l'équivalent-génération est celle de Boichard et al (1997). Si, par exemple, à la $7^{\text {ème }}$ génération du pedigree d'un individu, on connaît seulement 41 ancêtres (éventuellement répétés) sur les 128 possibles, cette génération ne compte que pour 0,32 (41/128). Le nombre d'équivalents-générations est le cumul de ce type de ratio entre la première et la dernière génération remontée.

\section{5 / Consanguinité et parenté des femelles}

L'augmentation de la consanguinité a été sensiblement linéaire sauf en 1992-1993 (figure 2). Le taux de consanguinité des génisses nées en 1999 est de l'ordre de 5 \%. Le taux d'accroissement annuel s'établit à 0,22\% soit davantage qu'il n'était prévu, en dépit d'un nombre plus important de taureaux (cf plus haut). Cependant, le développement de l'élevage des génisses et le rajeunissement régulier de leurs mères ont entraîné une succession des générations plus rapide que prévue, ce qui a pu biaiser les premiers calculs, cette

Tableau 5. Nombre moyen d'équivalents-générations connues en fonction de l'année de naissance.

\begin{tabular}{|l|c|c|c|c|c|c|}
\hline & 1978 & 1982 & 1986 & 1990 & 1994 & 1999 \\
\hline Femelles & 3,8 & 4,7 & 5,4 & 5,6 & 6,4 & 6,9 \\
\hline Mâles & 5,0 & 4,0 & 5,3 & 6,2 & 6,0 & 7,2 \\
\hline
\end{tabular}

Figure 2. Evolution de la consanguinité et de la parenté moyenne des femelles BPN nées de 1978 à 1999.

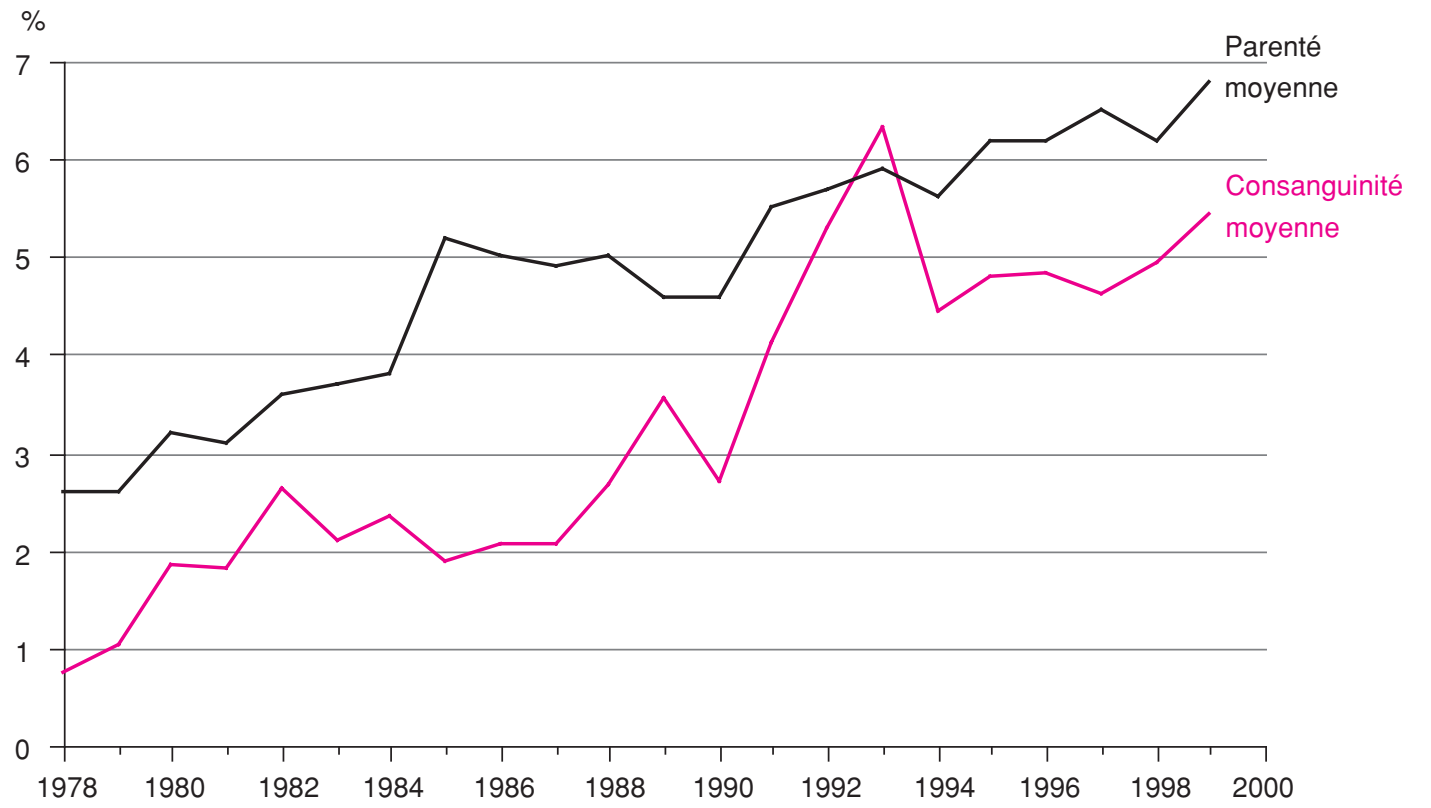

\section{Le taux}

de consanguinité a augmenté à peu près linéairement d'environ 0,22\% par an. 
fois dans un sens trop favorable. Le taux d'accroissement annuel du coefficient de parenté s'établit à $0,20 \%$. Le coefficient de parenté obtenu en 1999 est à peu près celui d'individus apparentés par deux grands-parents.

\section{6 / Origine des gènes des femelles}

Le tableau 6 montre le nombre d'ancêtres fondateurs (ou fondateurs, en abrégé) contribuant en probabilité au génome de la population. On y voit que les gènes peuvent provenir de 220 fondateurs mais qu'une vingtaine de ceux-ci suffisent à rendre compte de $50 \%$ des gènes présents dans la population. Mais de nombreuses origines seraient encore représentées avec une faible probabilité. Ainsi, pour les femelles nées en 1999, 211 origines pourraient être représentées. Au total, le nombre efficace de fondateurs pour les mêmes animaux est de l'ordre de 46.

Une appréciation plus sévère, mais plus réaliste, de la situation consiste à répertorier les origines effectivement représentées ( Crow et Kimura 1970, Boichard et al 1997). Etant donné les difficultés du calcul analytique pour des pedigrees complexes, on a simulé (500 répétitions indépendantes) la transmission aléatoire des gènes fondateurs le long de l'ensemble des pedigrees connus.

Pour les animaux nés en 1999, 6, 12, 17, 24, 32 fondateurs différents expliquent respectivement $25 \%, 50 \%, 75 \%, 90 \%, 100 \%$ des gènes effectivement représentés (tableau 7). En clair, 12 fondateurs fréquents sont à l'origine de la moitié des gènes de la population et 20 moins fréquents sont à l'origine de l'autre moitié. On note depuis 1978 un resserrement des origines et c'est pourquoi, une des futures tâches du plan rénové sera de renforcer la fréquence des gènes peu représentés. Pour résumer la situation, on parle aussi de nombre efficace de génomes : c'est le nombre de fondateurs indépendants qui, utilisés de manière égale, créerait la même variance génétique pour un caractère quantitatif que des fondateurs plus nombreux mais représentés de manière inégale (Crow et Kimura 1970, Boichard et al 1997). Sa valeur est de 8 pour les animaux nés en 1999. Il est vrai qu'avec ce type de calcul, la valeur obtenue en race Normande n'est que de ... 22 (Boichard et al 1997).

\section{7 / Accouplements rétrospectifs à parenté minimum}

La figure 3 montre les résultats d'un exercice rétrospectif extrêmement instructif, simulant des accouplements à parenté minimum. Dans les schémas fictifs 1 et 2 , les taureaux sont conservés tels quels avec leur pedigree réel Cependant, les vaches nées dans le plan sont issues d'accouplements fictifs entre ces taureaux et les vaches présentes. On considère les vaches présentes la première année du plan et les filles nées des inséminations correspondantes : ces filles réelles sont alors transformées en filles fictives identiques aux filles réelles sauf pour le père, celui-ci étant donné par le système d'accouplement à parenté minimum, pour l'année considérée. On réitère la même opération tous les ans jusqu'au temps présent. Les vaches fictives se substituent donc progressivement aux vaches réelles tout en conservant exactement la même carrière.

Dans le schéma fictif 1 , les taux d'utilisation des taureaux année par année (de naissance de leurs filles) sont identiques aux taux réels. Sous cette contrainte, un algorithme de calcul recherche les accouplements à parenté minimale entre les taureaux concernés et les vaches ayant donné des filles (d'après le fichier réel). Dans le schéma fictif 2, le taux d'utilisation des taureaux présents une année donnée est uniformisé.

L'efficacité des accouplements à parenté minimum est manifeste puisque les schémas 1 et 2 auraient conduit en 1999 à un coefficient de consanguinité de $3,4 \%$ et $2 \%$ respectivement (au lieu de 5,4\%). Cette simple constatation amène donc à revoir totalement la conception du programme.

Tableau 6. Nombre d'ancêtres fondateurs contribuant en probabilité au génome de la population femelle, en fonction de la date de naissance.

\begin{tabular}{|c|c|c|c|c|c|c|}
\hline & 1978 & 1982 & 1986 & 1990 & 1994 & 1999 \\
\hline $25 \%$ & 8 & 6 & 6 & 8 & 6 & 6 \\
$50 \%$ & 29 & 22 & 20 & 23 & 19 & 18 \\
$75 \%$ & 72 & 53 & 54 & 58 & 44 & 44 \\
$90 \%$ & 115 & 93 & 97 & 102 & 81 & 79 \\
$100 \%$ & 230 & 212 & 215 & 245 & 227 & 211 \\
\hline Nombre efficace de fondateurs & 70 & 55 & 46 & 62 & 50 & 46 \\
\hline
\end{tabular}

Tableau 7. Nombre d'ancêtres fondateurs contribuant en réalité au génome de la population femelle, en fonction de la date de naissance

\begin{tabular}{|c|c|c|c|c|c|c|}
\hline & 1978 & 1982 & 1986 & 1990 & 1994 & 1999 \\
\hline $25 \%$ & 17 & 11 & 8 & 11 & 10 & 6 \\
$50 \%$ & 31 & 20 & 15 & 17 & 15 & 12 \\
$75 \%$ & 51 & 29 & 23 & 23 & 21 & 17 \\
$90 \%$ & 63 & 36 & 31 & 32 & 29 & 24 \\
$100 \%$ & 71 & 42 & 38 & 39 & 37 & 32 \\
\hline Nombre efficace de fondateurs & 29 & 16 & 11 & 11 & 10 & 8 \\
\hline
\end{tabular}


Figure 3. Consanguinité moyenne des femelles BPN nées de 1978 à 1999 selon le plan d'accouplement réel ou deux plans fictifs (voir texte).

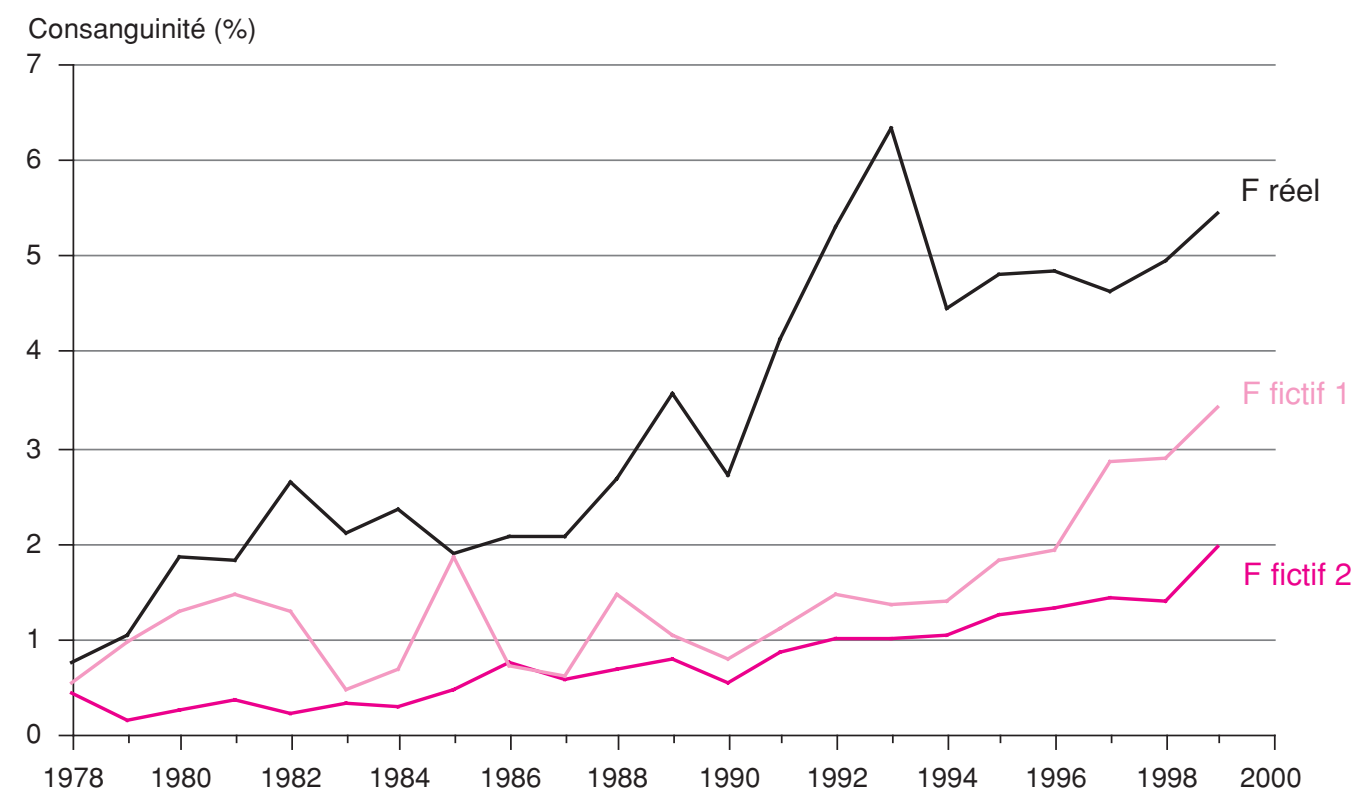

\section{3 / Le nouveau plan}

\section{1 / Grandes lignes}

Le nouveau plan part du constat que la conduite d'un schéma de conservation rotatif avec des groupes de reproduction (les familles) est extrêmement rigide et fragile visà-vis des dérives éventuelles. On préfère pour cette raison introduire un schéma plus souple et dynamique dans le temps, qui s'appuie à chaque instant sur la situation réelle existante pour optimiser les décisions. Plus précisément, on cherche chaque instant à minimiser la parenté moyenne entre les animaux (moyenne des couples pris deux à deux) ainsi que leur consanguinité propre. La prise en considération de la parenté moyenne ente animaux intègre à la fois la diversité des origines et la consanguinité de leur descendance conjointe. Le critère d'optimisation est donc à vocation multiple et les théoriciens ont montré qu'il permet de maximiser le nombre d'allèles fondateurs encore présents dans la population (Crow et Kimura 1970).

Le premier point essentiel du nouveau programme est l'utilisation d'accouplements à parenté minimale. Sous la contrainte que les taureaux d'IA disponibles sont utilisés en proportion égale sur la population (contrainte facilement réalisable quand le programme a atteint son régime de croisière), un algorithme de calcul sélectionne pour chaque vache le taureau présentant une parenté minimale avec elle (cf paragraphe 2.7, schéma fictif 2). Le second point essentiel est qu'on cherche à renforcer la fréquence des gènes rares pour éviter leur extinction totale, notamment au travers de la méthode de choix des mères à taureaux. Le troisième point essentiel est qu'on cherche à maximiser le nombre de taureaux d'IA disponibles la même année. Cette dernière orientation aura pour effet de tempérer l'augmentation de la parenté ou de la consanguinité. Elle permettra aussi de réduire l'aléa sur la valeur génétique moyenne des taureaux utilisés (vu les effectifs, l'efficacité des choix sur ascendance ou sur descendance est en effet limitée) et donc de mieux satisfaire les éleveurs d'une race en situation de relance, soucieux de nouveauté mais en l'absence de risques génétiques.

Comme dans l'ancien programme, chaque taureau est remplacé en rythme de croisière par un fils. Ce remplacement est programmé à la fin de la période d'utilisation de ce taureau. Théoriquement, il faudrait extraire d'une série de candidats issus d'accouplements programmés, ceux qui auraient l'impact le plus favorable dans le futur s'ils procréaient des génisses, à une époque où la composition de la population des futures conjointes n'est pas totalement certaine. On utilise donc une procédure simplifiée, quoiqu'encore relativement complexe. On choisit d'abord les femelles ayant les plus faibles coefficients moyens de parenté avec toute la population femelle (sous la réserve qu'elles aient au moins trois générations connues du côté maternel, la généalogie paternelle étant très bien connue), sans imposer leur appartenance à la population au contrôle laitier (donc contrairement au plan précédent). Leur effectif est proportionnel (environ multiplié par 15) au nombre de taureaux à remplacer chaque année. Les groupes de futures mères à taureaux sont ensuite alloués aux différents taureaux en cherchant la parenté minimale entre conjoints. On considère ensuite l'ensemble des mâles nés de ces accouplements et on choisit un seul mâle pour chaque père à taureaux. Dans ce but, on choisit parmi toutes les combinaisons possibles celle qui minimise l'ensemble des parentés : entre mâles retenus d'une part, entre mâles retenus et femelles présentes d'autre part. En effet, celles-ci seront en grande partie impliquées dans les accouplements concernant ces taureaux. Tous ces calculs sont effectués en mettant en œuvre une méthode rapide qui donne directement les parentés moyennes (Colleau 2002) sans passer par le calcul individuel de chaque coefficient de parenté. 


\section{2 / Simulation}

L'objectif de la simulation est d'apprécier à long terme (30 ans) les conséquences globales des nouvelles mesures envisagées. Il s'agit de simulations aléatoires avec génération d'animaux fictifs (descendants des animaux actuels) suivant le protocole précédent. La simulation est indispensable car la prédiction analytique relative à l'évolution de la parenté et de la consanguinité dans un cas aussi complexe est particulièrement difficile et en tout cas ne figure pas dans la littérature scientifique. En particulier, les accouplements ne sont pas aléatoires mais programmés dynamiquement. Il faut par ailleurs tenir compte du fait que les conséquences de l'ancien système influencent la mise en place du nouveau. Pendant longtemps, le 'régime de croisière' ne peut donc être atteint, hypothèse très souvent retenue dans la littérature parce qu'elle permet une formulation analytique relativement simple.

Vu l'importance cruciale de ce point pour l'application concrète du nouveau programme, on cherche à préciser la politique idéale d'utilisation et de renouvellement des taureaux d'IA. On simule donc quatre scénarios dont le premier (scénario A) correspond approximativement à la continuation de la politique actuelle.

- Scénario A : un taureau nouveau par an utilisé pendant 10 ans

- Scénario B : deux taureaux nouveaux par an utilisés pendant 5 ans

- Scénario C : deux taureaux nouveaux par an utilisés pendant 10 ans

- Scénario D : quatre taureaux nouveaux par an utilisés pendant 5 ans

En phase de croisière, les nombres de taureaux disponibles chaque année sont donc de
10, 10, 20 et 20 respectivement pour les 4 scénarios. Etant donné la situation de départ, les scénarios $\mathrm{B}, \mathrm{C}, \mathrm{D}$ amènent dés le début de leur application à remplacer les taureaux par plusieurs fils. De manière à limiter l'effet des aléas de simulation concernant les animaux fictifs, chaque scénario est répété indépendamment 10 fois en dépit de la lourdeur des calculs qu'entraîne la reproduction de toutes les étapes de la gestion génétique. Dans tous ces schémas, la procédure décrite plus haut est appliquée de façon identique.

Un élément important est que ces calculs sont effectués dans un contexte de croissance démographique, puisqu'on utilise les paramètres démographiques observés rétrospectivement La stabilité de ces paramètres dans le futur est bien sûr hypothétique mais nous n'avons pas encore d'indication permettant de la mettre en doute. Le tableau 8 montre les effectifs moyens observés au cours des simulations.

Le tableau 9 indique les coefficients de consanguinité observés chez les jeunes génisses de l'année. Un élément marquant est la notable diminution des coefficients de consanguinité en 2005, par rapport aux valeurs de 1999 : ils sont en effet réduits approximativement de moitié. C'est essentiellement le résultat de la pratique d'accouplements à parenté minimale. Vu la longueur de l'intervalle entre générations, les populations mâle et femelle n'ont en effet pas encore substantiellement changé. Pour la même raison, il n'y a pas de différences appréciables suivant les schémas. Les coefficients de consanguinité réaugmentent ensuite de $1 \%$ jusqu'en 2020 , soit une augmentation annuelle de $0,07 \%$ entre 2005 et 2020. On constate que dans cette période, les différences entre schémas ne sont pas encore très nettes. Elles finissent par apparaître ensuite car les augmentations annuelles de consan-

Tableau 8. Effectifs moyens mis en jeu dans les simulations (vaches et génisses de l'année).

\begin{tabular}{|l|c|c|c|c|c|c|}
\hline \multicolumn{1}{|c|}{ Année } & 2005 & 2010 & 2015 & 2020 & 2025 & 2030 \\
\hline Vaches & 1206 & 1369 & 1565 & 1710 & 1971 & 2190 \\
Génisses & 153 & 177 & 200 & 222 & 247 & 270 \\
\hline
\end{tabular}

Tableau 9. Taux de consanguinité (\%) des génisses selon l'année de naissance.

\begin{tabular}{|c|c|c|c|c|c|c|}
\hline Scénario & 2005 & 2010 & 2015 & 2020 & 2025 & 2030 \\
\hline A & 2,6 & 3,4 & 3,4 & 3,6 & 4,5 & 4,7 \\
\hline B & 2,7 & 3,0 & 3,3 & 3,8 & 4,2 & 4,7 \\
\hline C & 2,7 & 3,2 & 3,4 & 3,7 & 4,0 & 4,5 \\
\hline D & 2,6 & 3,0 & 3,3 & 3,6 & 4,0 & 4,2 \\
\hline
\end{tabular}

Tableau 10. Nombre d'ancêtres fondateurs contribuant à des proportions croissantes du génome de la population femelle née en 2030.

\begin{tabular}{|c|c|c|c|c|}
\hline & A & B & C & D \\
\hline $25 \%$ & 9 & 9 & 9 & 9 \\
$50 \%$ & 26 & 27 & 26 & 27 \\
$75 \%$ & 59 & 64 & 60 & 62 \\
$90 \%$ & 103 & 113 & 103 & 106 \\
$100 \%$ & 220 & 219 & 219 & 224 \\
\hline Nombre efficace de fondateurs & 71 & 73 & 71 & 75 \\
\hline
\end{tabular}


guinité sont alors de $0,11 \%, 0,09 \%, 0,08 \%$ et $0,06 \%$ pour les scénarios A, B, C et D respectivement. On note cependant que dans tous les cas, les niveaux de consanguinité sont encore notablement inférieurs à ceux de 1999. Ce résultat est essentiel et justifie finalement la mise en ouvre du nouveau plan.

Le tableau 10 indique le nombre de fondateurs espérés contribuant au génome de la population des femelles nées en 2030. On note dans tous les scénarios une réaugmentation du nombre de fondateurs représentés, de telle sorte que la structure des origines (déséquilibre de la représentation des origines) rappelle en 2030 celle observée en 1978. Pour donner une image concrète de la nouvelle situation, on peut remarquer que les ancêtres majeurs de 1999 qui intervenaient à hauteur de $26 \%$ de l'origine des gènes n'interviennent plus que pour $18,2 \%, 18,4 \%, 18,8 \%$ et $18 \%$ pour les scénarios A, B, C et D.

\section{4 / Discussion et conclusion}

La situation génétique actuelle du programme BPN a été évaluée à partir de pedigrees généralement connus en profondeur $(6,9$ générations en moyenne pour les femelles nées en 1999 et 24 générations au maximum), nettement plus que dans les 11 autres programmes bovins (Danchin-Burge et Avon 2000) où ces valeurs vont de 2,5 à 4,4 , de 7 à 13 respectivement, ce qui empêche de faire une comparaison vraiment objective avec la situation de ces races. Par exemple, les animaux récents de la race Villard-de-Lans atteignent un coefficient moyen de consanguinité de $4,2 \%$, mais le nombre d'équivalents-générations connus n'est que de 4,2. On doit rappeler que tous ces programmes sont à la fois plus récents et de taille plus réduite que le programme BPN.

Le programme de conservation devrait normalement conduire à une sensible amélioration de la situation existante. Le changement de politique d'accouplement est le fait majeur dont les effets dominent sur environ 20 ans (période pendant laquelle le comportement de la consanguinité est nettement curvilinéaire). Par ailleurs, l'érosion du nombre de lignées d'origine devrait normalement cesser.

Néanmoins, la pente ultime de la consanguinité en fonction du temps est très clairement liée à la politique concernant les taureaux. Un effort tout particulier devra être consenti pour augmenter le nombre de taureaux rentrant annuellement en service (certainement au moins 2, scénario C). Cela est bien illustré par l'application de la prédiction de Hill (1972 et 1979 ) aux 4 scénarios précédents qui amène à des valeurs d'accroissement annuel de la consanguinité de 0,38\%, $0,32 \%, 0,19 \%$ et $0,16 \%$ respectivement. La hiérarchie des scénarios apparaît bien. Les valeurs absolues sont bien plus élevées que celles indiquées par la simulation entre 2020 et 2030, essentiellement parce que cette prédiction suppose que les accouplements sont aléatoires, ce qui n'est pas le cas (Caballero 1994). Un effort supplémentaire devrait être entrepris pour retarder la mise en service des taureaux, ce qui augmenterait l'intervalle de génération sur les voies mâles (père-fils et père-fille) et, toutes choses étant égales par ailleurs, diminuerait les rythmes annuels d'augmentation du taux de consanguinité. Si les taureaux étaient mis en service à $7 \mathrm{ans}$, les valeurs précédentes seraient de $0,23 \%$, $0,17 \%, 0,12 \%$ et $0,09 \%$ respectivement et seraient donc pratiquement réduites de moitié. Là encore, cette amélioration suppose non seulement qu'elle soit bien acceptée par les éleveurs, mais aussi certaines capacités financières car, pendant la phase de transition, on récolterait annuellement beaucoup plus de taureaux qu'on n'en ferait entrer en service. Sur ce même plan de conservation, Chevalet et de Rochambeau (1985) ont recommandé l'utilisation de la monte naturelle avec un schéma rotatif. Le nouveau plan conserve toujours l'insémination artificielle comme outil de reproduction, considérant que dans une structure d'élevages à effectifs faibles, et très dispersés géographiquement, les avantages de l'IA l'emportent largement sur ses inconvénients.

Finalement, la gestion optimisée des accouplements grâce à la prise en compte des parentés obtenues par le calcul se révèle être une voie d'approche intéressante pour la conservation des petites populations. Il n'y a aucune raison de penser qu'elle ne puisse pas convenir aussi à d'autres populations que la population Bretonne Pie-Noire.

Il est fréquemment indiqué que la sélection pour l'hétérozygotie de marqueurs neutres associée à des procédures classiques comme la sélection intra-famille est une mesure efficace pour freiner l'accroissement de la consanguinité réelle (Toro et al 1988 et1998, Verrier et Rognon 2000, Wang et Hill 2000). Cette supériorité par rapport aux méthodes mettant en œuvre les seuls pedigrees dépend bien entendu du nombre de marqueurs utilisés. Cependant, par simple réalisme financier, les potentialités d'une version 'moléculaire' du nouveau programme n'ont pas été testées. En effet, le typage de 200 nouvelles génisses par an pour seulement un marqueur par chromosome coûterait déjà 20000 euros soit l'équivalent de la mise en service de 10 nouveaux taureaux par an (5000 doses par taureau).

On peut faire la même remarque pour la transplantation embryonnaire, technique trop coûteuse malgré des avantages certains. On pourrait grâce à elle multiplier les familles en danger d'extinction dans la population et donc gagner sur deux tableaux : celui de l'entretien de la diversité génétique et celui de la maîtrise de la consanguinité. 


\section{Références}

Audiot A, 1995. Races d'hier pour l'élevage de demain. INRA éditions, Paris, 229 p.

Boichard D., Maignel L., Verrier E., 1997. The value of using probabilities of gene origin to measure genetic variability in a population. Genet. Sel. Evol., 29, 5-23.

Bougler J., 1974. Conséquences générales de la disparition des races. Ethnozootechnie Numéro spécial Races domestiques en péril, 1-40.

Brossard G., 1999. Vers une relance de la race bovine Bretonne Pie-Noir. Mémoire Inst. Sup. Agr. de Beauvais, 80 p.

Caballero A., 1994. Developments in the prediction of effective population size. Heredity, 73, 657-679.

Caballero A., Toro M.A., 2000. Interrelations between effective population size and other pedigree tools for the management of conserved populations. Genet. Res. Camb., 75, 331-343.

Chevalet C., De Rochambeau H., 1985. Predicting the genetic drift in small populations. Livest. Prod. Sci., 13, genetic $207-218$.

Colleau J.J., 2002. An indirect approach to the extensive calculation of relationship coefficients. Genet. Sel. Evol. (à paraître).

Colleau J.J., Krychowski T., Le Borgne M., 1976. Programme de conservation de la Bretonne Pie-Noir. Document ronéoté, $15 \mathrm{p}$

Crow J.F., Kimura M., 1970. An introduction to population genetics theory. Burgess publishing company, Minneapolis, USA

Danchin-Burge C., Avon L., 2000. Analyse de la variabilité génétique de races bovines à très petits effectifs, après vingt ans d'actions de conservation. Renc. Rech. Ruminants, 7, 145-148.

De Rochambeau H., Fournet-Hanocq F., Vu Tien Khang J. 2000. Measuring and managing genetic variability in small populations. Ann. Zootech., 49, 77-93.

Grosclaude F., Aupetit R.Y., Lefebvre J., Mériaux J.C., 1990. Essai d'analyse des relations génétiques entre les races bovines françaises à l'aide du polymorphisme biochimique. Genet. Sel. Evol., 22, 317-338.

Henry C., 1992. Bilan de 15 ans de sauvegarde de la race Bretonne Pie Noir. Mémoire Inst. Sup. Agr. de Beauvais, 78 p.

Hill W.G., 1972. Effective size of populations with overlapping generations. Theor. Pop.Biol., 3, 278-289.

Hill W.G., 1979. A note on effective population size with overlapping generations. Genetics, 92, 317-322.

Kimura M., Crow J. F, 1963. On the maximum avoidance of inbreeding. Genet. Res., 4, 399-415.
Le Borgne M., 1977. La Bretonne Pie-Noire, mise en place d'un programme de sauvegarde. Mémoire Inst. Sup. Agr. de Beauvais, $50 \mathrm{p}$.

Moureaux S., Boichard D., Verrier E., 2000. Utilisation de l'information généalogique pour l'estimation de la variabilité génétique de huit races bovines laitières française d'expression nationale ou régionale. Renc. Rech. Ruminants, 7, 149-152

Quéméré P., 1981. La race bovine Bretonne Pie-Noir Bilan des actions de sauvegarde 1975-1981. Journées du 10 et 11 décembre 1981. Société des éleveurs B.P.N., 10 p.

Quéméré P., Bertrand G., 1976. Une race en voie de disparition : la Bretonne Pie-Noir. Enquête de situation, premiers essais de sauvegarde. UNLG, $63 \mathrm{p}$.

Quéméré P., Colleau J.J., Krychowski T., 1978. Bilan des actions de sauvegarde en race bovine bretonne Pie-noir. Bull. Tech. Dep. Genet. Anim. INRA, 26, 49-58.

Quéméré P., Flageul L. Goenvec C., 1989. Evaluation d'un programme de sauvegarde et documentation sur la race conservée: le cas de la race bovine Bretonne Pie-Noir. race conservée : le cas de la race bovine Bretonne Pie-Noir. Colloque sur la gestion des ressources génétiques des espèces animal
B.R.G., 212-224.

Quéméré P., Colleau J.J., Henry C., Sergent J., 1992 Actions et acteurs, difficultés et ébauches de solutions dans la conduite d'un programme de sauvegarde d'une race en péril. Cas de la race bovine Bretonne Pie-Noir. Journée d'étude du 24 Septembre 1992 au CEZ Rambouillet (DRAC, Ministère de l'Agriculture), 16 p.

Quéméré P., Bougler J., Brossard, G., Sergent J. 1999. La race bovine bretonne Pie-noire (BPN) : de la sauvegarde à la relance. Renc. Rech. Ruminants, 6, 43-46.

Sanson A., 1884. Traité de zootechnie. IV Zoologie et zootechnie spéciales : Bovidés taurins et bubalins (3ème ed) Libr. Agric. Maison Rustique, Paris

Toro M.L., Nieto B., Salgado C., 1988. A note on minization of inbreeding in small scale breeding programmes. Livest. Prod. Sci., 20, 317-323.

Toro M.L., Silio J., Rodriganez J., Rodriguez C., 1998. The use of molecular markers in conservation programmes of live animals. Genet. Sel. Evol., 30, 585-600.

Verrier E., Rognon X., 2000. Utilisation des marqueurs pour la gestion de la variabilté génétique des populations. INRA Prod. Anim., numéro hors-série 'Génétique moléculaire : principes et application aux populations animales', 253-257.

Vissac B. 1972. Une seconde révolution de l'élevage. Sciences et Avenir, 309, 896-901.

Wang J.L., Hill W.G., 2000. Marker-assisted selection to increase effective population size by reducing mendelian segregation variance. Genetics, 154, 475-489.

Wright S., 1921 Systems of mating. Genetics 6, 111-143.

\begin{abstract}
Genetic management of the Breton BlackPied cattle breed.

Since its conservation programme started in $\mathbf{1 9 7 6}$, the Breton Black-Pied cattle breed has been one of the twelve French dairy breeds under conservation. A profile of corresponding breeders was briefly outlined and informations are given about the population size over time. The guidelines used for past genetic management are presented. Usual criteria for monitoring genetic variability are calculated and discussed (coancestry, inbreeding and number of founding genes still pre-
\end{abstract}

Abstract sent). The motivations and principles of a future breeding plan, expected to be more efficient, are presented. The trend observed in the current programme would lead to an average inbreeding coefficient of about 10 $11 \%$ for animals born in 2030 . Simulations showed that implementing the new plan would reduce this coefficient to only $4.5-5 \%$.

COLLEAU J. J., QUÉMÉRÉ P., LARROQUE H., SERGENT J., WAGNER C., 2002. Gestion génétique de la race bovine Bretonne Pie-Noire : bilan et perspectives. INRA Prod. Anim., 15, 221-230. 